Math Modeling. Numerical Experiment Математическое моделирование. Численный эксперимент

DOI: 10.17516/1999-494X-0365

УДК $539.3+539.4$

\title{
Strength of Curvilinearly Reinforced Plates in a Polar Coordinate System
}

\author{
Yuri V. Nemirovsky a and Natalia A. Feodorova*b \\ ${ }^{a}$ Institute of Theoretical and Applied Mechanics \\ named after S. A. Khristianovich, SB RAS \\ Novosibirsk, Russian Federation \\ ${ }^{b}$ Siberian Federal University \\ Krasnoyarsk, Russian Federation
}

Received 21.09.2021, received in revised form 15.10.2021, accepted 21.11.2021

\begin{abstract}
A computer simulation of flat structures to create a fiber composite with curved reinforcement along spiral trajectories in the polar coordinate system has been performed. Numerical comparisons of the reachability of limit states for various reinforcement structures by two families of continuous curved fibers have been made. The calculations take into account the influence of a constant temperature field. In this paper, analytical solutions of the reinforcement intensity function of the differential equation of the constancy of the fiber cross sections for the families of logarithmic and algebraic spirals are found. The analysis of the results shows that due to the choice of curved laying of reinforcing fibers, it is possible to obtain a structure with predetermined properties.
\end{abstract}

Keywords: structural model, curved reinforcement trajectories, thermoelastic behavior, structure limit state.

Acknowledgements. This work was carried out with partial financial support from the Russian Foundation for Basic Research (project No. 19-01-00038) and partial support from a grant provided by the Russian Foundation for Basic Research, the Government of the Krasnoyarsk Territory, and the Regional Science Foundation No. 20-41-240002.

Citation: Nemirovsky, Yu.V., Feodorova, N. A. Strength of curvilinearly reinforced plates in a polar coordinate system, J. Sib. Fed. Univ. Eng. \& Technol., 2021, 14(8), 952-964. DOI: 10.17516/1999-494X-0365

(C) Siberian Federal University. All rights reserved

This work is licensed under a Creative Commons Attribution-Non Commercial 4.0 International License (CC BY-NC 4.0).

* Corresponding author E-mail address: feodorova.natalia@mail.ru 


\title{
Прочность криволинейно армированных пластин
}

\section{В полярной системе координат}

\author{
Ю.В. Немировскийа ${ }^{a}$ Н.А. Федорова ${ }^{\sigma}$ \\ ${ }^{a}$ Институт теоретической и прикладной механики \\ им. С. А. Христиановича СО РАН \\ Российская Федерация, Новосибирск \\ ${ }^{6}$ Сибирский федеральныий университет \\ Российская Федерация, Красноярск
}

\begin{abstract}
Аннотация. В работе выполнено компьютерное моделирование плоских конструкций по созданию волокнистого композита с криволинейным армированием вдоль спиралевидных траекторий в полярной системе координат. Проведены численные сравнения достижимости предельных состояний для разнообразных структур армирования двумя семействами непрерывных криволинейных волокон. В расчетах учтено влияние постоянного поля температур. Для семейств логарифмических и алгебраических спиралей найдены аналитические решения функции интенсивности армирования дифференциального уравнения постоянства сечений волокон. Анализ результатов показывает, что за счет выбора криволинейной укладки армирующих волокон можно получить конструкцию с заранее заданными свойствами.
\end{abstract}

Ключевые слова: структурная модель, криволинейные траектории армирования, термоупругость, предельное состояние конструкции.

Благодарности. Работа выполнена при частичной финансовой поддержке Российского фонда фундаментальных исследований (проект № 19-01-00038) и частичной поддержке за счет средств гранта, предоставленного РФФИ, Правительством Красноярского края, Краевым фондом науки № 20-41-240002.

Цитирование: Немировский, Ю.В. Прочность криволинейно армированных пластин в полярной системе координат / Ю. В. Немировский, Н. А. Федорова // Журн. Сиб. федер. ун-та. Техника и технологии, 2021, 14(8). С. $952-964$. DOI: $10.17516 / 1999-494 X-0365$

\section{Введение}

Проблемы создания эффективных в эксплуатации тонкостенных конструкций актуальны для объектов авиационной, аэрокосмической, судостроительной и машиностроительной техники. Для конструкций из однородных материалов эти проблемы получили широкое развитие при создании теории оптимального проектирования и сводятся к поиску законов профилирования толщины конструкции вдоль ее отсчетной поверхности. Поскольку использование однородных материалов, как показывают эти исследования, не позволяет существенно повышать эксплуатационные качества создаваемых изделий, в последние десятилетия разрабатываются многочисленные технологические приемы (сварка взрывом, склейка, плазменное и газодинамическое напыление, армирование, профильное фрезерование и др.), которые дают возможность создавать композитные конструкции без каких-либо ограничений на характер перераспределения объединяемых материалов и их физико-механические характеристики.

Вопрос создания эффективных композитных конструкций при этом сводится не только к геометрическому профилированию конструкций, но также к отбору и установлению необходимого местоположения составляющих изделие конструкционных материалов, позволяющего 
наиболее эффективно использовать положительные качества всех материалов или большинства из них. Такая проблема была определена как проблема гибридного или мозаичного проектирования в цикле работ Ю. В. Немировского и его учеников, например $[1,2]$. Для некоторых простых конструкций важные перспективные результаты получены в работах авторов [3-5].

В условиях плоского напряженного состояния эффективные конструкции могут быть созданы путем армирования по определенным криволинейным траекториям с послойным чередованием изотропных и армированных структур. Такие комбинированные конструкции открывают большие возможности для управления напряженно деформированным состоянием и создания высокоэффективных конструкций. Уровень современных технологий позволяет на практике получать криволинейно армированные плоские конструкции. Численные эксперименты дают рекомендации для создания типов армированных конструкций с прогнозируемыми свойствами.

\section{Постановка задачи}

В серии исследований авторов работы выполнено математическое моделирование армированной вдоль криволинейных траекторий плоской конструкции на основе структурной модели композита Ю. В. Немировского. В работах [3-5] на основе структурной модели композита в рамках линейной неоднородной анизотропной задачи упругости получена разрешающая система дифференциальных уравнений, описывающая поведение армированной кольцевой пластины. Система сформулирована относительно радиального и окружного перемещения в полярной системе координат. Система и граничные условия представляют собой двухточечную краевую задачу неканонического вида. Построен эффективный численный метод, учитывающий специфику задачи [5]. На его основе в настоящей статье проводится анализ численных результатов. Входные данные задачи представлены в табл. 1, 2.

В табл. 2 использованы обозначения: $E, v$ - соответственно модуль Юнга и коэффициент Пуассона материала связующего; $E_{1}, E_{2}$ - модули Юнга материала первого и второго семейств

Таблица 1. Краевая задача в обезразмеренных переменных

Table 1. Boundary value problem in dimensionless variables

\begin{tabular}{|l|l|l|l|}
\hline \multicolumn{1}{|c|}{ Тип армирования } & $\begin{array}{c}\text { Формулировка } \\
\text { краевой задачи } \\
\text { в обезразмеренных } \\
\text { переменных }\end{array}$ & \multicolumn{1}{c|}{$\begin{array}{c}\text { Выбор материала } \\
\text { связущего и волокон }\end{array}$} & $\begin{array}{l}\text { Выбор относительных } \\
\text { размеров конструкции } \\
{\left[R_{1}, 1\right]}\end{array}$ \\
\hline $\begin{array}{l}\text { Два семейства } \\
\text { армирующих волокон }\end{array}$ & Жесткая заделка & $\begin{array}{l}\text { Алюминий }(\mathrm{Al}) \\
\text { со стальными } \\
\text { волокнами. } \\
\text { Титан }(T i) \\
\text { с керамическими } \\
\text { волокнами }\end{array}$ & $\begin{array}{l}1 . R_{1}=0,25 \\
2 . R_{1}=0,1 \\
3 . R_{1}=0,5\end{array}$ \\
\hline $\begin{array}{l}\text { Два семейства } \\
\text { армирующих волокон }\end{array}$ & Кручение & $\begin{array}{l}\text { Алюминий }(A l) \\
\text { со стальными } \\
\text { волокнами. } \\
\text { Титан }(T i) \\
\text { с керамическими } \\
\text { волокнами }\end{array}$ & $\begin{array}{l}1 . R_{1}=0,25 \\
2 . R_{1}=0,1 \\
3 . R_{1}=0,5\end{array}$ \\
\hline
\end{tabular}


Таблица 2. Механические параметры армированной круговой пластины [6]

Table 2. Mechanical parameters of the reinforced circular plate [6]

\begin{tabular}{|c|c|c|c|c|c|c|c|}
\hline № & $v$ & $\begin{array}{c}E,(T i) \\
\text { ГПА }\end{array}$ & $E,(A l)$ & $\begin{array}{c}E_{1}=E_{2} \\
\text { ГПА } \\
\text { (керамика) }\end{array}$ & $\begin{array}{c}E_{1}=E_{2} \\
\text { ГПА } \\
\text { (сталь) }\end{array}$ & $\omega_{01}$ & $\omega_{02}$ \\
\hline 1 & 0,3 & 94 & 70 & 350 & 200 & 0,3 & 0,3 \\
\hline 2 & 0,3 & 94 & 70 & 350 & 200 & 0,05 & 0,376 \\
\hline 3 & 0,3 & 94 & 70 & 350 & 200 & 0,1 & 0,318 \\
\hline 4 & 0,3 & 94 & 70 & 350 & 200 & 0,51 & 0,18 \\
\hline
\end{tabular}

волокон; $\omega_{01}, \omega_{02}$ - начальные интенсивности армирования на внутреннем контуре, определяемые способом изготовления композита.

Проверка условий разрушения упругого армированного материала имеет свои особенности. В работах $[3,7]$ показано, что необходимо проверять условие прочности связующего и условие прочности арматуры.

Для численных расчетов проведено обезразмеривание: относительный линейный размер кольцевой пластины имеет интервал $\left[R_{1}, 1\right]$, силовые характеристики относятся к модулю Юнга материала семейств армирующих волокон, в расчетах выбрано отношение к модулю Юнга $E_{1}$. Мы используем обезразмеренные переменные и критерий прочности Мизеса - Баландина для неоднородного материала запишем в форме

$$
\left(\sigma_{R}^{c}\right)^{2}+\left(\sigma_{b \theta}^{c}\right)^{2}-\sigma_{R}^{c} \sigma_{b \theta}^{c}+3\left(\sigma_{R \theta}^{c}\right)^{2}+\left(\sigma_{c}^{-}-\sigma_{c}^{+}\right)\left(\sigma_{R}^{c}+\sigma_{b \theta}^{c}\right)<\sigma_{c}^{-} \sigma_{c}^{+}
$$

В (1) материал изотропного связующего имеет различные пределы прочности при растяжении $\sigma_{c}^{+}$и сжатии $\sigma_{c}^{-}$. Обезразмеренные напряжения $\sigma_{R}^{c}, \sigma_{b \theta}^{c}, \sigma_{R \theta}^{c}$ в связующем через деформации $\varepsilon_{\rho}, \varepsilon_{\theta}, \varepsilon_{\rho \theta}$ и интенсивности армирования $\omega_{m}$ в полярной системе координат $(\rho, \theta)$ вычисляем по формулам

$$
\begin{aligned}
& \sigma_{R}^{c}=\frac{1}{E_{1}}\left(\frac{\Omega E}{1-v^{2}}\left(\varepsilon_{\rho}+v \varepsilon_{\theta}-\alpha^{c}(1+v) T\right)+\sum_{1}^{m} \sigma_{m} \omega_{m} \cos ^{2} \varphi_{m}\right), \\
& \sigma_{b \theta}^{c}=\frac{1}{E_{1}}\left(\frac{\Omega E}{1-v^{2}}\left(\varepsilon_{\theta}+v \varepsilon_{\rho}-\alpha^{c}(1+v) T\right)+\sum_{1}^{m} \sigma_{m} \omega_{m} \sin ^{2} \varphi_{m}\right), \\
& \sigma_{R \theta}^{c}=\frac{1}{E_{1}}\left(\frac{\Omega E}{(1+v)} \varepsilon_{\rho \theta}+\sum_{1}^{m} \sigma_{m} \omega_{m} \cos \varphi_{m} \sin \varphi_{m}\right), \Omega .=1-\sum \omega_{m} .
\end{aligned}
$$

Здесь $\Omega$ - удельная интенсивность прослоек связующего между армирующими слоями.

Обезразмеренные напряжения $\sigma_{m}$ в $m$-м семействе армирующих волокон с углом армирования $\varphi_{m}$ примут вид

$$
\sigma_{m}=\frac{E_{m}}{E_{1}}\left(\varepsilon_{\rho} \cos ^{2} \varphi_{m}+\varepsilon_{\theta} \sin ^{2} \varphi_{m}+\varepsilon_{\rho \theta} \cos \varphi_{m} \sin \varphi_{m}+\alpha_{m}^{a} T\right)
$$

где $T$ - постоянная температура; $\alpha^{c}, \alpha_{m}^{a}-$ коэффициенты линейного температурного расширения материала связующего и материала $m$-го семейства волокон соответственно. 
Для семейств армирующих волокон предполагаем, что пределы прочности (текучести) $m$ го семейства при растяжении $\sigma_{m}^{+}$и сжатии $\sigma_{m}^{-}$различны. Непрерывные семейства армирующих волокон остаются упругими, если выполняются неравенства [7]

$$
-\frac{1}{E_{1}} \sigma_{m}^{-}<\frac{E_{m}}{E_{1}}\left(\varepsilon_{\rho} \cos \varphi_{m}^{2}+\varepsilon_{\rho \theta} \cos \varphi_{m} \sin \varphi_{m}+\varepsilon_{\theta} \sin \varphi_{m}^{2}\right)<\frac{1}{E_{1}} \sigma_{m}^{+} .
$$

Для проверки прочности армированного материала необходимо анализировать условия (1) и (3): условие на прочность материала связующего и условие на прочность (текучесть) семейств армирующих волокон.

На основании вышеизложенного следует ввести понятие «предельное упругое состояние» в некоторой точке рассматриваемой конструкции, по достижении которого хотя бы в одной точке, либо в связующем, либо в волокне, происходит выход за пределы упругости (напряжение превышает предел прочности или текучести). В данной точке может возникнуть микроразрушение. Уравнениями «модели», сформулированными в рамках теории упругости, уже не можем пользоваться. Происходит выход за рамки линейной теории упругости.

Левую часть критерия прочности (1) назовем функцией Баландина, обозначим ее как $S(R)$, будем определять и визуализировать ее значения для различных типов криволинейных структур армирования.

\section{Определение функции интенсивности армирования $\omega_{m}$}

Интенсивности армирования $\omega_{m}$ и углы армирования $\varphi_{m}$ связаны уравнениями, отражающими условие постоянства сечений волокон $[3,8]$, что обусловлено технологией изготовления волокнистого композита. В полярной системе координат $(\rho, \theta)$ уравнения имеют вид

$$
\frac{\partial}{\partial \rho}\left(\rho \omega_{m} \cos \varphi_{m}\right)+\frac{\partial}{\partial \theta}\left(\rho \omega_{m} \sin \varphi_{m}\right)=0
$$

Интенсивность армирования $\omega_{m}$ определяется как решение начальной задачи для уравнений (4). Приведем аналитические решения для интенсивности армирования на примерах рассматриваемых структур армирования.

1. Пусть дано семейство логарифмических спиралей вида $\rho=C b^{\theta}, C$ - параметр семейства, $b$ - параметр спирали, при $b>1$ спираль развертывается вокруг полюса против хода часовой стрелки, если $b<1$, то спираль закручивается по часовой стрелке. Вычислим угол армирования $\operatorname{tg} \varphi_{1}=\frac{\rho}{\rho^{\prime}}=\frac{1}{\ln b}$, то есть для логарифмической спирали угол армирования - некоторая константа. Определим интенсивность армирования $\omega_{1}(\rho)$ из уравнения (4), в нем производную по $\theta$ вычислим по формуле $\frac{\partial}{\partial \theta}=\frac{\partial \rho}{\partial \theta} \frac{\partial}{\partial \rho}$ с учетом уравнения траектории $\rho=C b^{\theta}$. В результате имеем

$$
\frac{\partial}{\partial \rho}\left(\rho \omega_{1}\right)+\rho \frac{\partial}{\partial \rho}\left(\omega_{1}\right)=0
$$


В (5) частную производную заменяем обычной производной по $\rho$, так как исключили зависимость от окружной координаты. Интегрирование (5) с учетом заданной интенсивности армирования $\omega_{0}$ на внутреннем контуре $\rho=\rho_{1}$ дает соотношение

$$
\omega_{1}=\frac{\omega_{0} \sqrt{\rho_{1}}}{\sqrt{\rho}} .
$$

2. Выполним армирование по семействам спиралей Архимеда. Спираль Архимеда задается уравнением $\rho=a \theta, a-$ коэффициент пропорциональности. Угол армирования находим из со-

отношения $\operatorname{tg} \varphi=\theta=\frac{\rho}{a}$, вычисляем $\sin \varphi, \cos \varphi$ через $\operatorname{tg} \varphi$, после подстановки в (5) получаем следующее уравнение для интенсивности армирования $\omega_{1}(\rho)$

$$
\frac{\partial}{\partial \rho} \frac{\rho \omega_{1}}{\sqrt{1+\operatorname{tg}^{2} \varphi}}=0
$$

так как оператор дифференцирования по окружной координате вдоль траектории армирования равен $\frac{\partial}{\partial \theta}=a \frac{\partial}{\partial \rho}$. Тогда интенсивность армирования в произвольной точке кольцевой пластины найдем по формуле

$$
\omega_{1}=\frac{C \sqrt{1+\operatorname{tg}^{2} \varphi}}{\rho} .
$$

Пусть на внутреннем контуре $\rho=\rho_{1}$ задан угол вхождения арматуры $\varphi_{0}$ и задана начальная интенсивность армирования $\omega_{0}$, что соответствует условиям технологического процесса. После вычисления константы интегрирования из условий на внутреннем контуре пластины получим

$$
\omega_{1}=\frac{\omega_{0} \sqrt{\rho_{1}^{2}+\rho^{2} \operatorname{tg}^{2} \varphi_{0}}}{\rho \sqrt{1+\operatorname{tg}^{2} \varphi_{0}}} .
$$

Для тангенса угла армирования по спирали Архимеда получим выражение

$$
\operatorname{tg} \varphi=\frac{\rho \operatorname{tg} \varphi_{0}}{\rho_{1}} .
$$

3. Армирование вдоль семейства траекторий «спицы велоколеса». «Спицы велоколеса» в полярной системе координат представляют семейство прямых, заданных уравнением

$$
\rho=\frac{a}{\sin \theta}
$$

где $a$ - константа, параметр велоколеса, $-\pi<\theta<0, \theta \neq \frac{\pi}{2}$. Вычислим $\rho_{\theta}^{\prime}$ найдем $\theta$ через $\rho$ из уравнения траектории:

$$
\theta=\arcsin \frac{a}{\rho},
$$

получим выражение тангенса угла армирования $\operatorname{tg} \varphi=-\operatorname{tg} \theta$ через полярный радиус

$$
-957-
$$




$$
\operatorname{tg} \varphi= \pm \frac{a}{\sqrt{\rho^{2}-a^{2}}}
$$

Оператор дифференцирования по окружной координате вдоль траектории армирования равен

$$
\frac{\partial}{\partial \theta}=-\frac{a \cos \theta}{\sin ^{2} \theta} \frac{\partial}{\partial \rho}
$$

В результате условие постоянства сечений волокон примет вид

$$
\frac{\partial}{\partial \rho}\left(\omega_{1} \sqrt{\rho^{2}-a^{2}}\right)+\rho \sqrt{\rho^{2}-a^{2}} \frac{\partial}{\partial \rho}\left(\frac{\omega_{1}}{\rho}\right)=0
$$

Пусть $\rho_{1}-$ внутренний радиус кольцевой пластины, $\theta_{0}$ - заданный угол выхода, тогда параметр «велоколеса» определяется выражением $a=\rho_{1} \sin \theta_{0}$. Общее решение (6) запишем

$$
\omega_{1}=\frac{C_{1} \sqrt{\rho}}{\left(\rho^{2}-\left(\rho_{1} \sin \theta_{0}\right)^{2}\right)^{\frac{1}{4}}},
$$

с учетом условия на внутреннем контуре $\left.\omega_{1}\right|_{\rho=\rho_{1}}=\omega_{0}$ получим интенсивность армирования по семействам траекторий «спицы велоколеса»

$$
\omega_{1}=\frac{\omega_{0} \sqrt{\rho}\left(\rho_{1}^{2}-\left(\rho_{1} \sin \theta_{0}\right)^{2}\right)^{\frac{1}{4}}}{\sqrt{\rho_{1}}\left(\rho^{2}-\left(\rho_{1} \sin \theta_{0}\right)^{2}\right)^{\frac{1}{4}}} .
$$

\section{Результаты численных экспериментов}

В приведенных ниже численных расчетах рассмотрены два семейства армирующих волокон $(m=1,2)$ из одинакового материала, поэтому при вычислении относительных силовых характеристик выполняем деление на модуль Юнга материала волокна $E_{1}$. При числовом анализе предельного упругого состояния используем следующие относительные (после отношения к модулю Юнга волокна) значения пределов прочности и текучести (правая часть критерия (1)):

1) квадрат относительного предела текучести для связующего алюминий при армировании стальными волокнами в соответствии с данными табл. 2 составляет $0,49 \cdot 10^{-6}$;

2) квадрат относительного предела текучести для связующего материала титан при армировании керамическими волокнами в соответствии с данными табл. 2 составляет $1,44 \cdot 10^{-6}$;

3) квадрат относительного предела текучести для связующего материала титан при армировании борными волокнами в соответствии с данными табл. 2 составляет 0,1296·10-6;

4) квадрат относительного предела текучести для связующего сталь (предел прочности стали от 700 МПА до 1970 МПА) при армировании семействами волокон из бериллия принадлежит интервалу $5,08 \cdot 10^{-6}-4,03 \cdot 10^{-6}$;

5) относительный предел прочности для стальных волокон при наименьшем значении предела прочности из данных табл. 2 составляет $3,5 \cdot 10^{-3}$;

6) относительный предел прочности для керамических волокон для данных табл. 2 составляет $5,7 \cdot 10^{-3}$.

Введем краткие обозначения для вида структуры армирования вдоль двух семейств непрерывных криволинейных траекторий кольцевой пластины. Способ армирования вдоль семей-

$$
-958-
$$


ства логарифмических спиралей и спиралей Архимеда обозначим $(\mathrm{A}+\mathrm{L})($ рис. $1 \mathrm{a}) ;$ с семейство спиралей Архимеда и семейства «спицы велоколеса» $(\mathrm{A}+\mathrm{V})($ рис. $1 b)$; семейства логарифмических спиралей и им изогональные семейства траекторий [9] - (L+Iz) (рис. 1в); семейства логарифмических спиралей и семейства «спицы велоколеса»-(L+V) (рис. 1г).
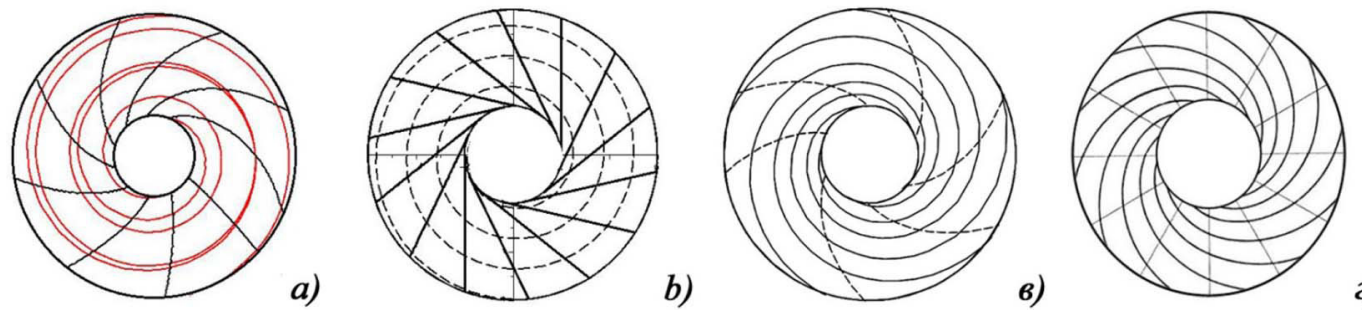

Рис. 1. Примеры армирования непрерывными семействами криволинейных волокон

Fig. 1. Examples of reinforcement with continuous curved fiber families

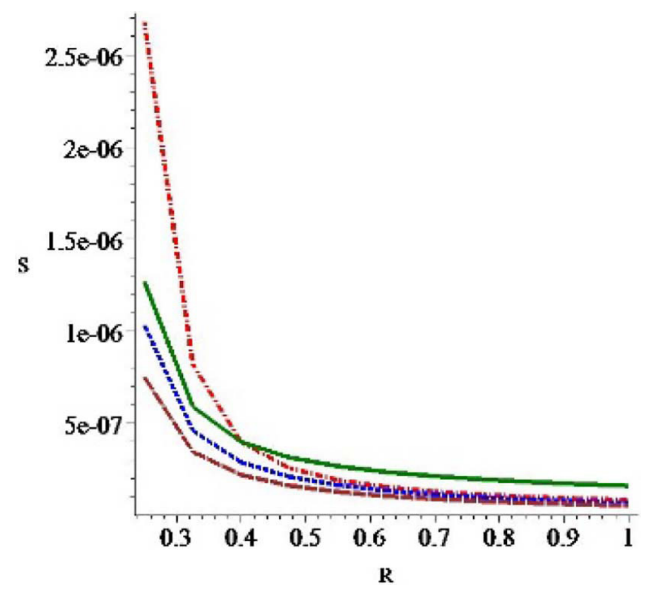

Рис. 2. Функция Баландина $S(R)$ для структуры армирования $(\mathrm{A}+\mathrm{L})$

Fig. 2. Balandin function $S(R)$ for the reinforcement structure $(\mathrm{A}+\mathrm{L})$

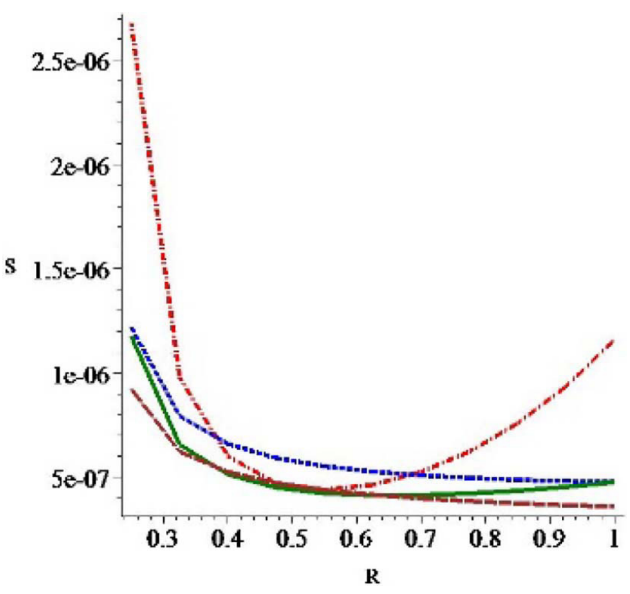

Рис. 3. Функция Баландина $S(R)$ для структуры армирования $(\mathrm{A}+\mathrm{V})$

Fig. 3. Balandin function $S(R)$ for the reinforcement structure $(\mathrm{A}+\mathrm{V})$

Фиксируем нагрузку в 2 МПА, рассмотрим краевую задачу для жесткой заделки, выберем материал связующего алюминий, армирование выполним стальными волокнами. На графиках рассмотрим для указанных структур (рис. 1) четыре варианта начальной интенсивности выхода арматуры на внутреннем контуре, представленных в табл. 2. На рисунках выводим четыре типа графиков 1 - сплошная линия (green), 2 - линия, состоящая из тире (brown), 3 - линия, состоящая из точек (navy), 4 - линия, состоящая из точек-тире (red). Результаты сравниваем по материалам и начальным интенсивностям армирования $\omega_{01}, \omega_{02}$ первого и второго семейств армирующих волокон, остальные геометрические параметры остаются фиксированными.

На рис. 2-5 изображены значения функции Баландина $S(R)$ для перечисленных наборов параметров связующего и семейств армирующих волокон (табл. 2). Сравнение между структура- 


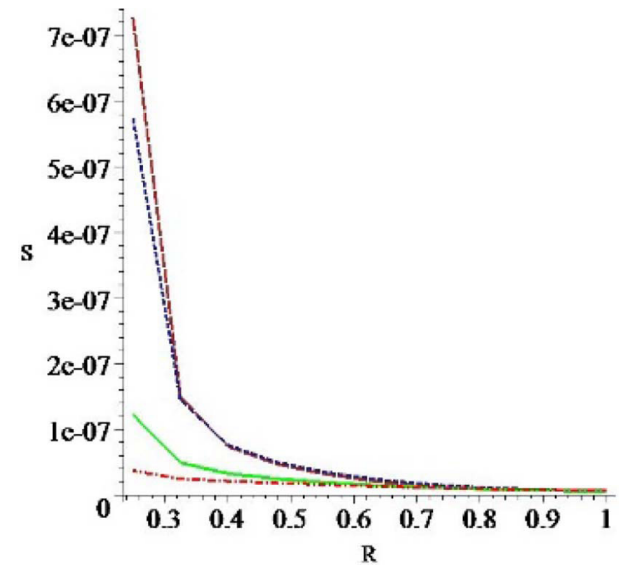

Рис. 4. Функция Баландина $S(R)$ для структуры армирования $(\mathrm{L}+\mathrm{Iz})$

Fig. 4. Balandin function $S(R)$ for the reinforcement structure $(\mathrm{L}+\mathrm{Iz})$

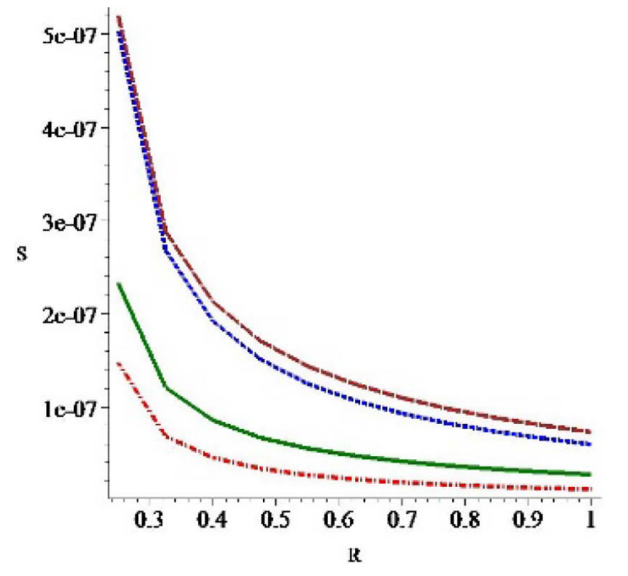

Рис. 5. Функция Баландина $S(R)$ для структуры армирования $(\mathrm{L}+\mathrm{V})$

Fig. 5. Balandin function $S(R)$ for the reinforcement structure $(\mathrm{L}+\mathrm{V})$

ми показывает, что выбор структур $(\mathrm{L}+\mathrm{Iz})$ и $(\mathrm{L}+\mathrm{V})$ не приводит к выходу за пределы упругости, в то время как $S(R)$ для $(\mathrm{A}+\mathrm{L})$ превышает относительный предел текучести для значений $\omega_{01}=$ $0,51, \omega_{01}=0,18$. Сравнение по материалам связующего и волокон демонстрирует, что функции Баландина $S(R)$ для кольцевой пластинки из титана с керамическими волокнами на порядок меньше $S(R)$ для пластинки из алюминия со стальными волокнами.

Серия графиков на рис. 6-11 показывает поведение осредненных напряжений в связующем $\sigma_{R}, \sigma_{\theta}$. Материал пластины - алюминий со стальными волокнами. Как видим, зависимость напряжений от обезразмеренного радиуса $R$ различна для разных типов структур. Так, напряжение $\sigma_{\theta}$ для структуры (L+Iz) (рис. 8) принимает наибольшее значение на внутреннем

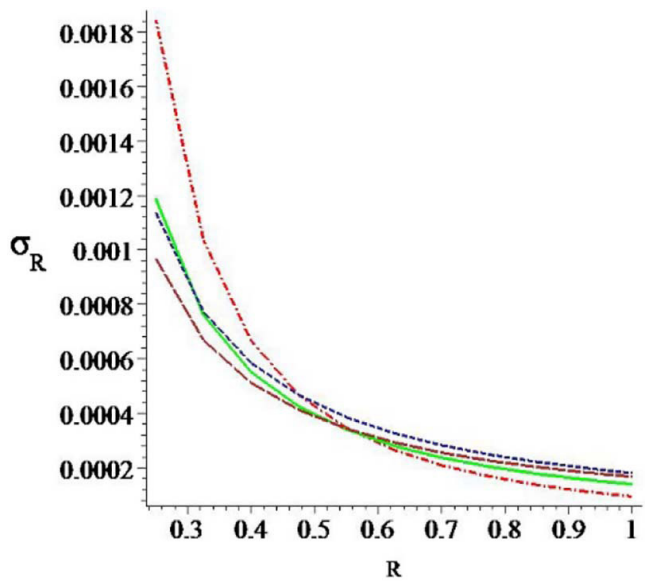

Рис. 6. Зависимость напряжения $\sigma_{R}$ от $R$ для структуры армирования $(\mathrm{A}+\mathrm{L})$

Fig. 6. Dependence of stress $\sigma_{R}$ on $R$ for the reinforcement structure $(\mathrm{A}+\mathrm{L})$

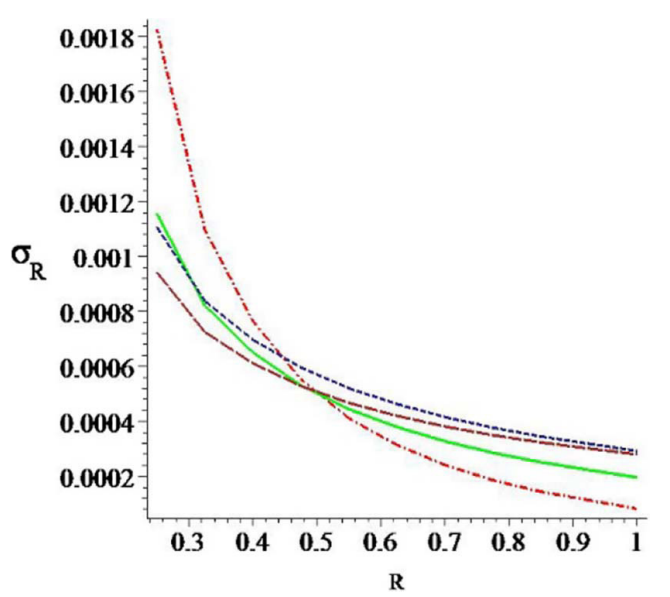

Рис. 7. Зависимость напряжения $\sigma_{R}$ от $R$ для структуры армирования $(\mathrm{A}+\mathrm{V})$

Fig. 7. Dependence of stress $\sigma_{R}$ on $R$ for the reinforcement structure $(\mathrm{A}+\mathrm{V})$ 


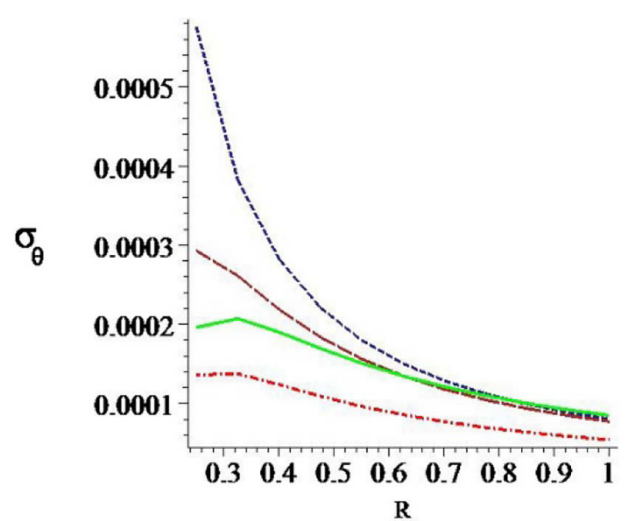

Рис. 8. Зависимость напряжения $\sigma_{\theta}$ от $R$ для структуры армирования (L+Iz)

Fig. 8. Dependence of stress $\sigma_{\theta}$ on $R$ for the reinforcement structure $(\mathrm{L}+\mathrm{Iz})$

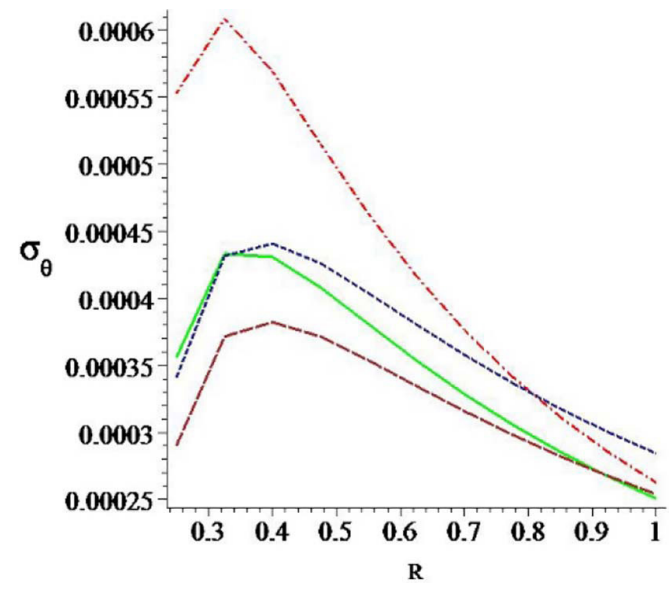

Рис. 10. Зависимость напряжения $\sigma_{\theta}$ от $R$ для структуры армирования $(\mathrm{A}+\mathrm{L})$

Fig. 10. Dependence of stress $\sigma_{\theta}$ on $R$ for the reinforcement structure $(\mathrm{A}+\mathrm{L})$

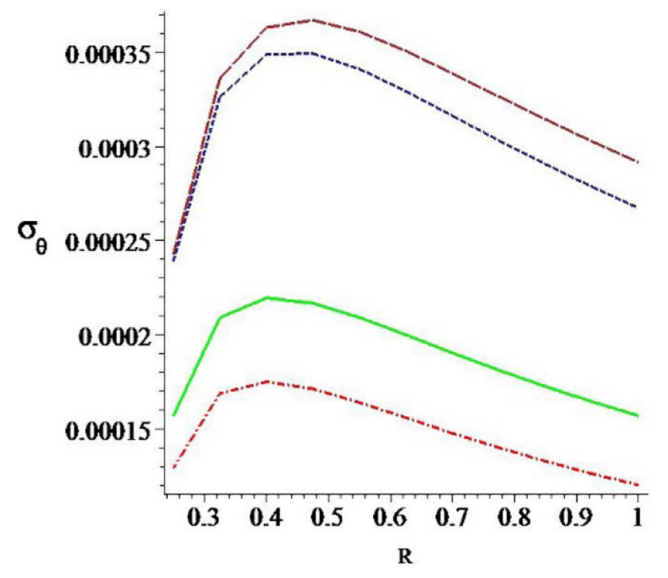

Рис. 9. Зависимость напряжения $\sigma_{\theta}$ от $R$ для структуры армирования (L+V)

Fig. 9. Dependence of stress $\sigma_{\theta}$ on $R$ for the reinforcement structure $(\mathrm{L}+\mathrm{V})$

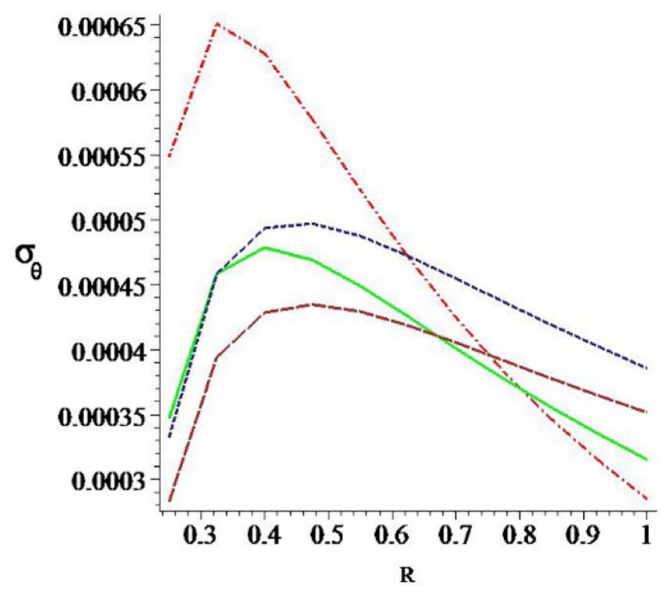

Рис. 11. Зависимость напряжения $\sigma_{\theta}$ от $R$ для структуры армирования (A+V)

Fig. 11. Dependence of stress $\sigma_{\theta}$ on $R$ for the reinforcement structure $(\mathrm{A}+\mathrm{V})$

контуре пластины, затем плавно убывает. Но для структур (L+V), (A+L), (A+V) это напряжение достигает наибольшего значения на относительном расстоянии 0,3 от внутреннего радиуса, затем убывает (рис. 9-11). Эти явления можно применять при создании волокнистого композита, посредством выбора структуры армирования учитывать специфику эксплуатации конструкции в реальных условиях.

В расчетах выполнено сравнение функции Баландина $S(R)$ при малых значениях начальной интенсивности армирования $\omega_{01}=0,01 ; \omega_{02}=0,01$. Все структуры с малой плотностью армирования показывают выход из зоны упругости, происходит нарушение условий (1), (2). Армирование же с плотностью $\omega_{01}=0,3 ; \omega_{02}=0,3$ дает возможность повышения амплитуды внешней нагрузки. Такие же результаты показывает анализ напряжений в волокне. Для слабой 
плотности армирования нарушается критерий (2). Все структуры с плотностью армирования 0,3 не достигают предельного состояния для данных материалов и рассмотренных условий нагружения.

На рис. 12-15 исследована зависимость функции Баландина для названных структур армирования $(\mathrm{A}+\mathrm{L}),(\mathrm{L}+\mathrm{Iz}),(\mathrm{L}+\mathrm{V}),(\mathrm{A}+\mathrm{V})$ после введения поля температур со значениями температуры (минус 40 , минус $20,20,40)$ градусов Цельсия $\left({ }^{\circ} \mathrm{C}\right)$. В $(2)$ в напряжениях, вызываемых полем температур, производится пересчет температуры в Кельвинах (К).

Характер зависимости функции Баландина с введением поля температур изменился, все значения $S(R)$ рис. $12-15$ на порядок больше по сравнению с $S(R)$ на рис. 2-5, максимальные

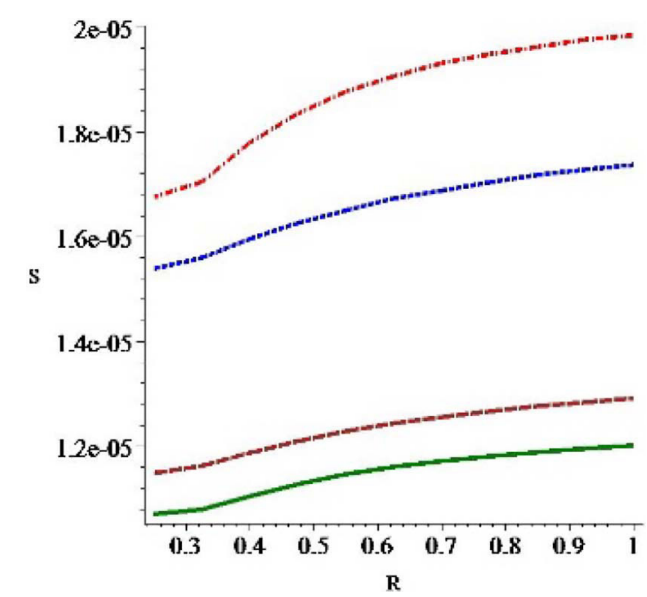

Рис. 12. Функция Баландина $S(R)$ для структуры армирования $(\mathrm{A}+\mathrm{L})$

Fig. 12. Balandin function $S(R)$ for the reinforcement structure $(\mathrm{A}+\mathrm{L})$

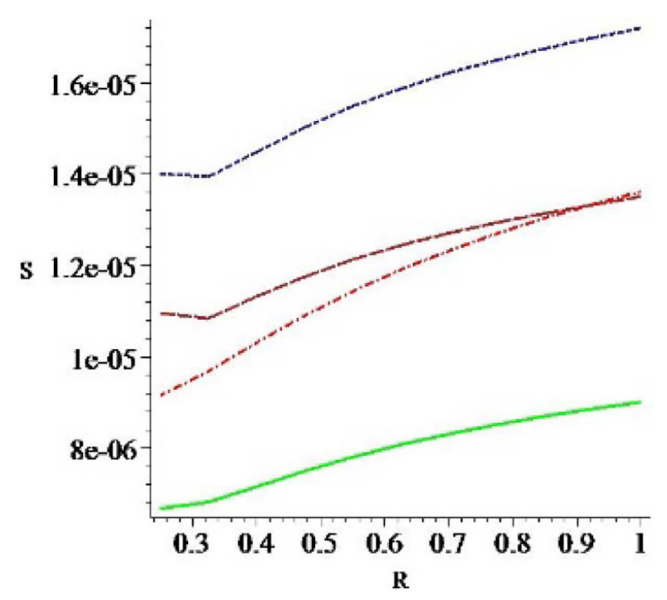

Рис. 14. Функция Баландина $S(R)$ для структуры армирования $(\mathrm{L}+\mathrm{Iz})$

Fig. 14. Balandin function $S(R)$ for the reinforcement structure $(\mathrm{L}+\mathrm{Iz})$

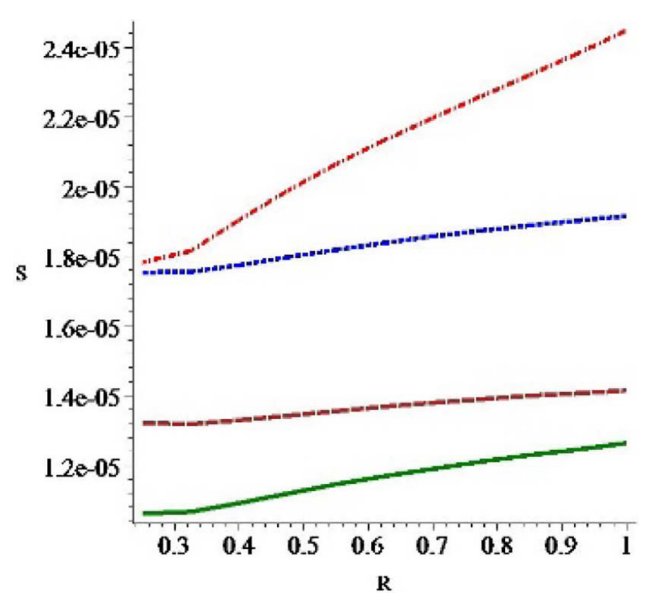

Рис. 13. Функция Баландина $S(R)$ для структуры армирования $(\mathrm{A}+\mathrm{V})$

Fig. 13. Balandin function $S(R)$ for the reinforcement structure $(\mathrm{A}+\mathrm{V})$

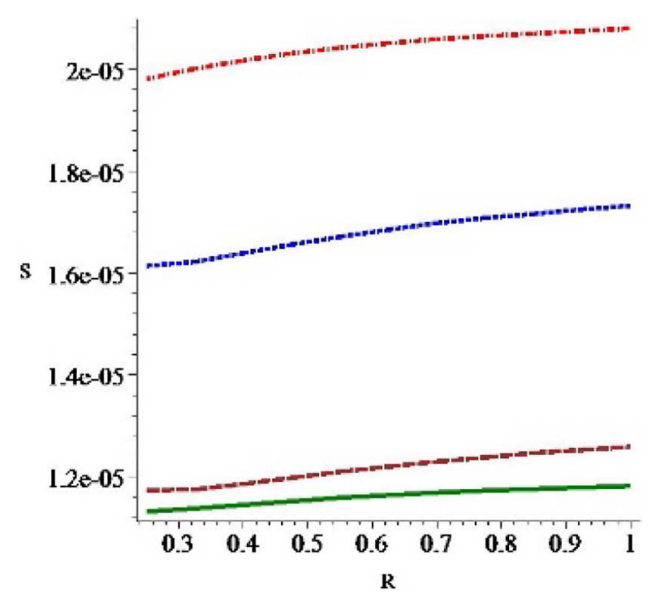

Рис. 15. Функция Баландина $S(R)$ для структуры армирования (L+V)

Fig. 15. Balandin function $S(R)$ for the reinforcement structure $(\mathrm{L}+\mathrm{V})$ 
значения $S(R)$ могут достигаться не обязательно на внутреннем контуре, что характерно было для рис. 2-5.

Анализ приведенных выше графиков позволяет сделать следующие выводы:

1. На величину напряжений в волокне и связующем влияет выбор материала связующего и армирующих волокон, что показано на графиках рисунков.

2. Введение на внутреннем контуре жесткой заделки приводит к существенному изменению уровня напряжений по сравнению с рассмотренными условиями ненулевых перемещений на внутреннем контуре кольцевой пластины.

3. На поведение напряжений в пластинке существенное влияние оказывает амплитуда внешней нагрузки.

4. Выбор величины внутреннего относительного радиуса кольцевой пластины оказывает влияние на уровень перемещений и, как показывают графики, значение функции Баландина на порядок отличаются для разных значений внутреннего радиуса.

5. Приведенные графики иллюстрируют существенное влияние геометрических параметров пластины (угол выхода арматуры на внутреннем контуре, начальная интенсивность армирования, выбор способа укладки непрерывных семейств армирующих волокон) на поведение напряжений в армированной пластине.

\section{Заключение}

Исследовано влияние изменения постоянного поля температур на прочность плоской конструкции, армированной вдоль двух непрерывных семейств криволинейных траекторий: семейство логарифмических спиралей и спиралей Архимеда, семейство логарифмических спиралей и им изогональных траекторий, семейство логарифмических спиралей и семейство «спицы велоколеса».

Расчеты показали широкие возможности управления свойствами армированной пластины за счет выбора геометрических параметров пластины. Меняя структуру армирования, получаем плоскую конструкцию с заранее заданными свойствами.

Уровень современных технологий позволяет на практике создавать криволинейно армированные плоские конструкции. Численные эксперименты дают рекомендации для создания типов армированных конструкций с прогнозируемыми свойствами.

\section{Список литературы / References}

[1] Немировский Ю.В., Янковский А.П. Мозаичное армирование плоских термоупругих конструкций с использованием различных критериев рационального проектирования. Механика композиционных материалов и конструкций, 2002, 8(3), 3-27 [Nemirovsky Yu.V., Yankovsky A.P. Mosaic reinforcement of flat thermoelastic structures using various criteria of rational design. Mechanics of composite materials and structures, 2002, 8(3), 3-27 (In Russian)]

[2] Amelina E.V., Golushko S.K., Yurchenko A.V. Analysis and design of hybrid pressure vessels. CEUR Workshop Proceedings, 2017, v. 1839, 244-257.

[3] Немировский Ю.В., Федорова Н.А. Математическое моделирование плоских конструкиий из армированных волокнистых материалов: СФУ, Красноярск, 2010, 136 с.

$$
-963-
$$


[Nemirovskij Yu.V., Feodorova N.A. Mathematical modeling of flat structures made of reinforced fiber materials, Krasnoyarsk: Sib. Fed. Univ. Publ., 2010, 136 p. (In Russian)]

[4] Немировский Ю.В., Федорова Н.А. Предельные деформации термоупругих плоских конструкций с криволинейным армированием. Вестник СибГАУ, 17(1), 2016, 73-78. [Nemirovsky Yu.V., Feodorova N.A. Limiting deformations of thermoelastic flat structures with curved reinforcement. Vestnik SibGAU, 17(1), 73-78 (In Russian)]

[5] Nemirovsky Yu.V., Feodorova N. A. Modeling limit states for curviliearly reinforced rotated disks. Mathematica Montisnigri, 2019, XLIV, 84-99.

[6] Васильев В.В., Протасов В.Д., Болотин В.В. и др. Композищионные материалы. Справочник. М.: Машиностроение, 1990. 510 с. [V.V. Vasil'ev, V.D. Protasov, V. V. Bolotin et al., Kompozicionnye materialy. Spravochnik, M.: Mashinostroenie, 1990, 510 p.]

[7] Немировский Ю.В., Резников Б.С. Прочность элементов конструкций из композитных материалов. Новосибирск: Наука, 1986. 165 с. [Nemirovskij Yu.V., Reznikov B. S. Strength of structural elements made of composite materials, Novosibirsk: Nauka, 1986, 165 p. (In Russian)]

[8] Бушманов С. Б., Немировский Ю. В. Оптимальное проектирование пластин при плоском напряженном состоянии. Прикл. механика и техн. физика, 1983, 5, 158-165 [Bushmanov S.B., Nemirovsky Yu. V. Optimal plate design under flat stress conditions. Prekl. mechanics and technology physics, 1983, 5, 158-165 (In Russian)]

[9] Федорова Н.А. Моделирование изогонально армированных кольцевых пластин в полярной системе координат. Журнал СФУ. Математика и физика 2011, 4(3), 400-405 [Feodorova N. A. Modeling isogonally reinforced annular plates in a polar coordinate system. J. Sib. Fed. Univ. Mathematics \& Physics 2011, 4(3), 400-405 (In Russian)] 